\title{
Integrating genomics to dig deeper into Wilms tumour biology
}

\author{
William Mifsud and Kathy Pritchard-Jones
}

Refers to Gadd, S. et al. A Children's Oncology Group and TARGET initiative exploring the genetic landscape of Wilms tumor. Nat. Genet. http://dx.doi.org/10.1038/ng.3940 (2017).

A new study provides a comprehensive catalogue of the genetic changes in Wilms tumour. Understanding the interplay between genetic and epigenetic aberrations during tumorigenesis and clonal evolution is fundamental to enabling prediction of who is at risk and which tumours will relapse when so many are curable.

Wilms tumour is the commonest paediatric renal malignancy. Improvements in chemotherapy, surgery, and radiotherapy, which is used in a minority of patients,now result in a $90 \%$ cure rate. However, predicting and treating tumour relapse remains difficult and a considerable number of patients might be overtreated or undertreated. Furthermore, even when cured of the original tumour, many patients have to contend with adverse treatment effects, including cardiac dysfunction, hepatotoxicity, secondary malignancies, and pregnancy complications, potentially compounded by their genetic susceptibility to renal failure, heart disease, and other tumours.

Over the past few decades, numerous studies aimed to elucidate the genetic events leading to Wilms tumour development. Discoveries have been tremendously accelerated by the application of massively parallel sequencing to large collections of Wilms tumour specimens from collaborative national and international studies. Three papers published in 2014 and 2015 expanded the repertoire of known Wilms tumour cancer genes from five to twelve ${ }^{3-5}$. In the most comprehensive genomic characterisation of Wilms tumours to date, Gadd et al. ${ }^{6}$ analysed a discovery set of 117 tumours (78 with favourable histology that relapsed and a large set of 39 high-risk, diffuse anaplastic tumours) using whole-genome sequencing in 80 tumours and whole-exome sequencing in 37 tumours, as well as mRNAseq and miRNAseq. In addition, the team tested a panel of 37 genes identified in the discovery set in 651 additional tumours by targeted capture sequencing: only around two-thirds of samples in this validation set had deleterious mutations in $\geq 1$ of these 37 genes, whereas genomewide sequencing of the discovery set found deleterious mutations in 115 of 117 (nearly all) tumours. Nevertheless, the results of this study expand the list of Wilms tumour cancer genes to $\geq 30$ genes. The challenge is that none of these genes individually accounts for tumour development in $>5 \%$ of patients.

The authors make an admirable attempt to reduce this complexity by mapping the discovered genes to a simplified set of developmental and molecular pathways. A number of genes identified that are recurrently mutated in Wilms tumour are specifically important in early nephrogenesis, whereas other genes have wide-ranging functions that include roles in nephrogenesis. Specifically, by performing parallel studies of mRNA and miRNA gene expression and methylation profiles, Gadd et al. ${ }^{6}$ find correlations with mutated genes that indicate different Wilms tumour subgroups. One group has features of a preserved nephrogenic state and another group predominantly has mutational and gene expression profiles indicating aberrant induction of the metanephric mesenchyme that normally triggers a mesenchymal-epithelial transition.

A subset of the tumours with a preserved nephrogenic state have mutations in the miRNA processing machinery, which are associated with overall reductions in miRNA levels, especially those of let-7a, which induces differentiation. By contrast, another group of tumours with a preserved nephrogenic state has similar miRNA expression profiles but no mutations in miRNA processing genes. The authors suggest that this discrepancy might be caused by copy number gain 
in LIN28B (which downregulates let-7a maturation) and/or loss of LET-7A. However, these copy number aberrations are not focal and, indeed, LIN28B copy number gain is a consequence of gain of the entire chromosome 6 in all cases. Hence, expression of miRNA processing genes might be affected directly by copy number changes.

In the tumours with aberrant induction, the researchers reviewed the known functions of the mutated genes and their regulatory interactions. They plausibly surmise that the aberrant induction is mediated by Wnt- $\beta$-catenin signalling (as expected from previous findings) and associated dysregulation of histone acetylation and transcriptional elongation, specifically through WNT9 and WNT4.

Notably, the clustering used to delineate the developmental and molecular pathways is not observed in tumours with diffuse anaplasia, which typically have mutations in TP53 and consequent genomic instability. This finding highlights a remaining challenge in the molecular characterisation of paediatric tumours in general. Tumours with considerable genomic instability, especially secondary to TP53 aberrations, have a predictable high risk of relapse; however, many relapsing tumours do not have TP53 mutations and seem to not have the same degree of genomic instability. Within the non-anaplastic clusters of Wilms tumour, detecting any molecular signature of relapse is particularly difficult. We suggest that genomic instability might be more difficult to detect when a tumour is sampled at a time when the clone with genomic instability has not yet taken over the entire tumour. Copy number changes and rearrangements are difficult to detect when they are subclonal in a tumour sample; indeed, controlling for sampling bias might be difficult, as all analyses depend on one small sample (at most a few $\mathrm{ml}$ ) from a large tumour mass (typically several hundred $\mathrm{ml}$ ). Alternatively, one can argue that the occurrence of tumours containing low levels of clones or only regional clones with considerable genomic instability is improbable, as these clones would rapidly outproliferate the rest of the tumour. We think that this argument might not apply in the context of a developmental tumour. In this setting, even the tumour precursor cells (nephrogenic rest cells) are typically proliferating rapidly at the time of tumorigenesis and, in a tumour characterised by proliferation and failure of differentiation of rapidly dividing progenitor cells, the growth advantage of cells with an unstable genome might be comparably small. Solving this problem will require analysis of multiple samples per tumour and improved methods of detecting subclonal structural genomic changes, such as genomic copy number changes, copyneutral loss of heterozygosity, and genomic rearrangements.

The study by Gadd et al. ${ }^{6}$ also confirms that many Wilms tumours have multiple tumorigenic genomic alterations. This finding and previous evidence of clonal evolution at copy number level ${ }^{7}$ set the foundation for future work that examines the temporal and spatial relationships and evolutionary trajectories of genomic events and tumour subclones, and whether and how these respond to treatment.

In Wilms tumour specifically, $37 \%$ of tumours have epimutations at the imprinted $11 \mathrm{p} 15$ locus, and an additional 32\% have paternal uniparental disomy (pUPD) at the same locus, both resulting in IGF2 overexpression and H19 expression $\operatorname{loss}^{8}$. pUPD is a very early, if not the earliest, event in those tumours harbouring it. pUPD was found in precursor nephrogenic rests ${ }^{9}$ and in all sampled parts of each tumour harbouring the change in a Wilms tumour multisampling study ${ }^{7}$. The $11 \mathrm{p} 15$ epimutation is possibly also a very early event. Studying the temporal and evolutionary relationship between this epigenetic event and the genetic mutations that might precede or follow it would be interesting.

Finally, this study confirms, much more comprehensively than before, a relatively high rate of germline mutations (10\%) that most probably predispose to Wilms tumour formation. Together with the previous finding that constitutional $11 \mathrm{p} 15$ abnormalities are present in $3 \%$ of patients with 
Wilms tumour ${ }^{10}$ and that mosaic postzygotic mutations might be difficult to detect, the real proportion of tumours that arise owing to an inherited mutation or an acquired mosaic mutation is likely to be higher. Future studies that systematically investigate this issue are required, for instance by comparing a patient's nontumorous cells with those of their parents. Improved understanding of this area should clarify the earliest events in Wilms tumour development and should also let us identify those patients at risk of subsequent malignancies or who have a raised risk of family members developing paediatric cancer. Studying the interplay of germline or mosaic predisposing mutations and epimutations at $11 \mathrm{p} 15$ and potentially other genomic loci might also be fascinating.

William Mifsud ${ }^{1,3}$ and Kathy Pritchard-Jones ${ }^{2,3}$ are at

Department of Histopathology $y^{1} \&$ Oncology$^{2}$, Great Ormond Street Hospital for Children, NHS Foundation Trust, Great Ormond Street, London, WC1N 3JH

${ }^{3}$ UCL Great Ormond Street Institute of Child Health, University College London, 30 Guilford Street, London, WC1N 1EH

Correspondence to K.P.-J. k.pritchard-jones@ucl.ac.uk

Competing interests statement

The authors have no competing interests to declare.

Pull quotes

"this study confirms ... a relatively high rate of germline mutations $(10 \%)$ that $\ldots$ predispose to Wilms tumour formation". 
1 Dome, J. S. et al. Children's Oncology Group's 2013 blueprint for research: renal tumors. Pediatr Blood Cancer 60, 994-1000, doi:10.1002/pbc.24419 (2013).

2 Brok, J., Treger, T. D., Gooskens, S. L., van den Heuvel-Eibrink, M. M. \& Pritchard-Jones, K. Biology and treatment of renal tumours in childhood. Eur J Cancer 68, 179-195, doi:10.1016/j.ejca.2016.09.005 (2016).

3 Torrezan, G. T. et al. Recurrent somatic mutation in DROSHA induces microRNA profile changes in Wilms tumour. Nat Commun 5, 4039, doi:10.1038/ncomms5039 (2014).

4 Walz, A. L. et al. Recurrent DGCR8, DROSHA, and SIX homeodomain mutations in favorable histology Wilms tumors. Cancer Cell 27, 286-297, doi:10.1016/j.ccell.2015.01.003 (2015).

5 Wegert, J. et al. Mutations in the SIX1/2 pathway and the DROSHA/DGCR8 miRNA microprocessor complex underlie high-risk blastemal type Wilms tumors. Cancer Cell 27, 298-311, doi:10.1016/j.ccell.2015.01.002 (2015).

6 Gadd, S. et al. A Children's Oncology Group and TARGET initiative exploring the genetic landscape of Wilms tumor. Nat Genet, doi:10.1038/ng.3940 (2017).

7 Cresswell, G. D. et al. Intra-Tumor Genetic Heterogeneity in Wilms Tumor: Clonal Evolution and Clinical Implications. EBioMedicine 9, 120-129, doi:10.1016/j.ebiom.2016.05.029 (2016).

8 Scott, R. H. et al. Stratification of Wilms tumor by genetic and epigenetic analysis. Oncotarget 3, 327-335, doi:10.18632/oncotarget.468 (2012).

9 Charles, A. K., Brown, K. W. \& Berry, P. J. Microdissecting the genetic events in nephrogenic rests and Wilms' tumor development. Am J Pathol 153, 991-1000, doi:10.1016/S0002-9440(10)65641-6 (1998).

10 Scott, R. H. et al. Constitutional 11p15 abnormalities, including heritable imprinting center mutations, cause nonsyndromic Wilms tumor. Nat Genet 40, 1329-1334, doi:10.1038/ng.243 (2008). 